\title{
High Incidence of Multidrug Resistant Pseudomonas aeruginosa Isolated from Infected Burn Wounds in a Tertiary Hospital
}

\author{
Romika Dawra*, Rajni Sharma, Rekha Bachhiwal and Aruna Vyas \\ Department of Microbiology, SMS Medical College, Jaipur, Rajasthan, India \\ *Corresponding author
}

\section{A B S T R A C T}

Keywords

MDR

P. aeruginosa,

Burn wound

Infection,

Antibiogram.

Article Info

Accepted:

20 January 2017

Available Online:

10 February 2017
Pseudomonas aeruginosa is an important cause of hospital acquired infection especially in patients admitted in critical care units such as intensive care units and burn care units. In recent times, it has emerged as a widespread Multi Drug Resistant (MDR) pathogen which requires antibiotic susceptibility testing on a regular as well as a periodic basis. This study was carried with an aim to determine the antimicrobial resistance pattern and prevalence of MDR $P$. aeruginosa infection among burns patients at a tertiary care center. This retrospective study was carried out from July 2015 to May 2016. In this study 938 non repetitive wound swabs from patients admitted in burn unit were collected and the isolates were identified by conventional phenotypic methods, the antibiotic sensitivity testing of all $P$. aeruginosa isolates was done using Kirby-Bauer disc diffusion method and the results were interpreted according to the Clinical and Laboratory Standards Institute guidelines. Out of these 938 clinical specimens, Pseudomonas aeruginosa was isolated in 392(41.79\%) samples. Out of these 392 isolates, $335(85.45 \%)$ were found to be MDR $P$. aeruginosa. $P$. aeruginosa showed maximum sensitivity to meropenem and imipenem (30.1\%) followed by levofloxacin (11.73\%), piperacillin-tazobactum (10.2\%). Carbenicillin, ceftazidime and cefoperazone were effective only in $1-5 \%$ isolates. Colistin was sensitive in $100 \%$ isolates.

\section{Introduction}

Pseudomonas aeruginosa is a leading cause of healthcare associated infection especially in patients admitted to critical care units such as intensive care units (ICUs) and burn care centers (Moazami-Goudarzi et al., 2012) Pseudomonas aeruginosa, a non-fermentative gram negative bacterium, is widely distributed in nature, including hospital environment. $P$. aeruginosa is a ubiquitous micro-organism that can rapidly acquire resistance to different broad-spectrum antibiotics (Indu et al., 2014). P. aeruginosa is responsible for about $10 \%-20 \%$ of nosocomial infections in the form of septicaemia in intensive-care units (ICUs), cystic fibrosis, burn and wound infections, etc., (Noha et al., 2015).

The immune suppression in burn patients in addition to the fact that $P$. aeruginosa has a 
predilection for moist and warm wound environments made it a major challenge for burn patients.

MDR $P$. aeruginosa phenotype is defined as a bacterium which is resistant to anti-microbial agents which is included in three or more anti-Pseudomonal anti-microbial classes (carbapenems, fluoroquinolones, penicillins /cephalosporins and aminoglycosides (Magiorakos et al., 2011). The mechanism of resistance in MDR $P$. aeruginosa is possibly through the production of several enzymes that inactivate beta-lactams and carbapenems such as extended spectrum beta lactamases (ESBLs) and metallo- $\beta$-lactamases (MBLs).

Pseudomonas aeruginosa is naturally resistant to penicillin and most of the b-lactam antibiotics. Carbapenems are the antibiotic of choice for treatment of $P$. aeruginosa infections. However, gradually increasing resistance to carbapenems has become a major concern. Resistance may be mediated by loss of the OprD porin, upregulation of multi-drug efflux pumps and production of certain b-lactamases and carbapenemases (Asghar, 2012; Pier et al., 2010).

Mechanisms of aminoglycoside resistance in $P$. aeruginosa are due to reduced uptake of aminoglycosides across the outer and cytoplasmic membranes associated with the production of aminoglycoside-modifying enzymes.

Modification of DNA gyrase is responsible for the development of resistance to quinolones. The use of polymyxins (polymyxin B and colistin) was limited due to their nephrotoxicity. However, emergence of resistance against most effective antipseudomonal agents has resulted in the need for use of these agents (Puneet et al., 2015). This study was conducted with an aim to determine the antimicrobial resistance pattern and prevalence of MDR $P$. aeruginosa infection among burns patients at a tertiary care center.

\section{Materials and Methods}

This retrospective study was conducted in the Dept of Microbiology from July 2015 to May 2016. The wound swabs from 938 patients admitted to the burn unit were collected and $P$. aeruginosa isolates were identified by conventional phenotypic methods for gram staining, colony morphology, motility, oxidase test, Hugh-Leifson oxidation fermentation test, susceptibility to polymyxin $\mathrm{B}$ and pyocyanin production. Other bacteria which were isolated were also processed and identified by standard microbiological techniques.

Antibiotic susceptibility testing was done on Mueller Hinton Agar according to CLSI guidelines by Kirby Bauer disk diffusion method. The antibiotic discs used are aztreonam (30ug), piperacillin (100ug) cefoperazone+sulbactam (75/30ug), ceftazidime (30ug), gentamicin (10ug), imipenem (10ug), piperacillin+tazobactum (100/10ug), tobramycin (10ug), meropenem (10ug), polymyxin B (300 units), colistin (10ug) and levofloxacin (5ug). All discs were procured from Hi-Media Pvt Ltd.

MDR $P$. aeruginosa was detected as a bacterium which was resistant to three or more anti-Pseudomonal antimicrobial classes (piperacillin+tazobactum, imipenem, ceftazidime and gentamicin).

Standard strain Pseudomonas aeruginosa ATCC27853 was used as a control.

\section{Results and Discussion}

Out of 938 wound swab specimens, the most common isolated organism was $P$. aeruginosa 
392/938 (41.79\%) followed by Escherichia coli 115/938 (12.2\%), Enterobacter cloacae 111/938 (11.8\%), Enterobacter aerogenes 101/938 (10.7\%), Proteus sp.80/938(8.5\%), Citrobacter sp. 49/938(5.2\%), Acinetobacter sp 36/938 (3.8\%), Staphylococcus aureus 26/938 (2.7\%) and CONS 21/938 (2.2\%). (TABLE 1)

Antimicrobial susceptibility testing was carried out for all 392 isolates of $P$. aeruginosa by Kirby -Bauer disk diffusion method. $P$. aeruginosa showed maximum sensitivity to meropenem and imipenem (30.1\%) followed by levofloxacin (11.73\%), piperacillin-tazobactum $(10.2 \%)$ Carbenicillin, ceftazidime and cefoperazone were effective only in 1-5\% isolates (Fig. 1).

According to the definition of MDR $P$. aeruginosa, 335 isolates out of $392(85.45 \%)$ were found to be MDR P. aeruginosa.

All the 392 isolates (100\%) were found to be susceptible to colistin.

Burn wound represents a susceptible site for opportunistic colonization by microorganisms of exogenous and endogenous origin.

Pseudomonas aeruginosa is a major pathogenic species in the family Pseudomonadaceae. $P$. aeruginosa is associated with colonization of otherwise healthy humans and animals It has the considerable potency to become resistant to many antibiotics and increasingly more antimicrobial resistant strains are being encountered in clinical practice, leaving the treating physician with limited treatment options that lead to a severe adverse outcome.

In the present study, the most prevalent organism isolated from burn patient was $P$. aeruginosa (41.79\%). Other studies such as
Arslan et al., (1999), Naqvi et al., (2005), Bhatt et al., (2015), Sharma et al., (2011) showed a prevalence of $P$. aeruginosa infection among burn patients to be $53.97 \%$, $59.6 \%, 54.9 \%$ and $61.95 \%$ respectively.

In the present study $85 \%$ isolates were found to be resistant to piperacillin-tazobactum combination. Similarly MozamiGoudarzi and Eftekhar (2012) have shown increased resistance to piperacillin-tazobactum combination (87.2\%) and Naqvi et al., (2005) showed $81.8 \%$ resistance. Similarly S Upadhya et al., (2014) showed $82.4 \%$ resistance to piperacillin-tazobactum combination whereas reports by Bhatt et al., (2015) and Sharma et al., showed 66\% resistance and $69.8 \%$ resistance respectively.

Among aminoglycoside 91\% isolates were resistant to gentamicin and $90 \%$ isolates were resistant to tobramycin. Similarly Naqvi et al., (2005) showed $93.2 \%$ resistance to gentamicin and $95.5 \%$ resistance to tobramycin. Bhatt et al., (2015) showed 84\% and $75 \%$ resistance to gentamicin and tobramycin respectively. However Upadhaya et al., (2014) showed $47.1 \%$ and $100 \%$ isolates to be resistant to gentamicin and tobramycin respectively.

Carbapenems are the antibiotics of choice for MDR $P$. aeruginosa infection but increasing resistance against carbapenems has now become a serious concern. In this study, it was found that $70 \%$ isolates were resistant to meropenem and imipenem. However Bhatt et al., (2015) showed resistance to meropenem and imipenem were 54\% and $61 \%$ respectively. Mozami -Gordarzi and Eftekhar in their study found $94.7 \%$ isolates resistant to both imipenem and meropenem. Similarly Upadhaya et al., (2014) showed $94.1 \%$ and $88.2 \%$ resistance to meropenem and imipenem respectively in their study. 
Table.1 Prevalence of organisms isolated from swab culture of burn wound (N=938)

\begin{tabular}{|l|c|c|}
\hline Organism & No of strain isolated & Percentage(\%) \\
\hline Pseudomonas aeruginosa & 392 & 41.7 \\
\hline Escherichia.coli & 115 & 12.2 \\
\hline Enterobacter.cloacae & 111 & 11.8 \\
\hline Enterobacter.aerogenes & 101 & 10.7 \\
\hline Proteus sp & 80 & 8.5 \\
\hline Citrobacter $s p$ & 49 & 5.2 \\
\hline Acinetobacter $s p$ & 36 & 3.8 \\
\hline Staphylococcus aureus & 26 & 2.7 \\
\hline $\begin{array}{l}\text { Coagulase negative } \\
\text { staphylococci }\end{array}$ & 21 & 2.2 \\
\hline
\end{tabular}

Fig.1 Antibiogram of Pseudomonas aeruginosa isolates

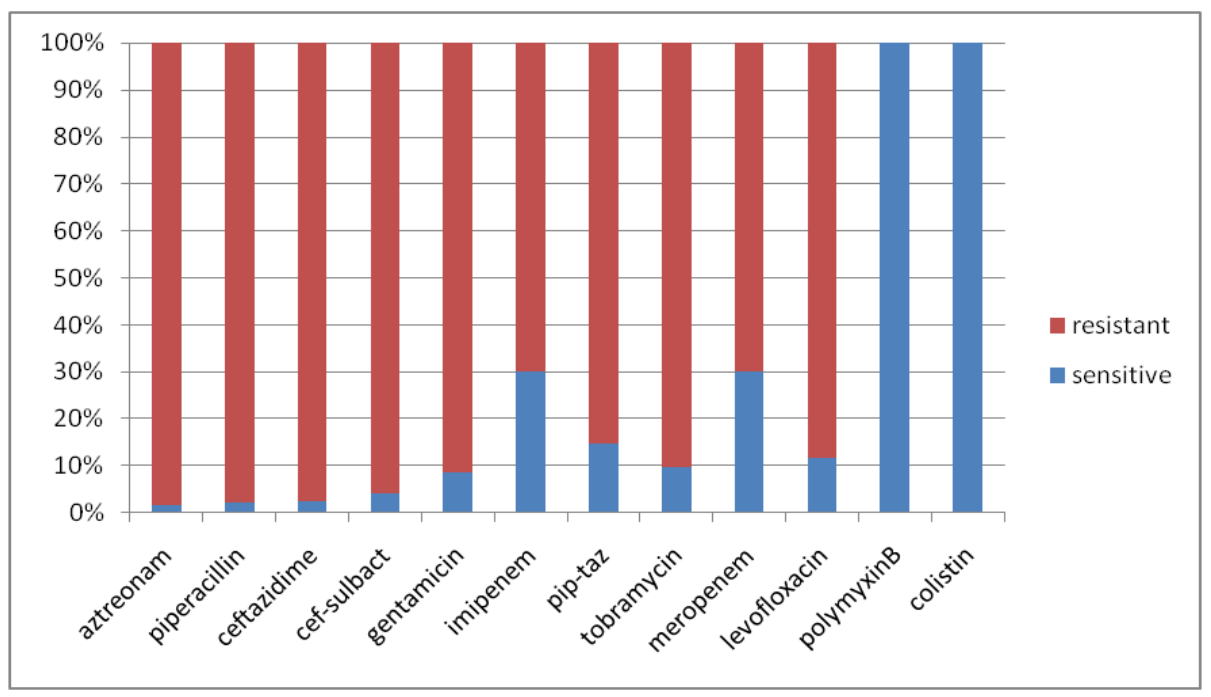

In this study, $85.45 \%$ isolates were found to be MDR, which is more than findings of Bhatt et al., (2015) who found $76.8 \%$ isolates to be MDR. Similarly Saderi et al., (2014) showed 69\% isolates to be MDR.However S Upadhaya et al., (2014) and MoazamiGoudarzi and Eftekhar (2012) showed 100\% MDR isolates in burns patients.

The absence of new anti-pseudomonal agents against MDR $P$. aeruginosa has amplified the problem. Therefore, polymyxin B and colistin are becoming the last resort for the treatment of such infections. Colistin should mainly be used as salvage therapy in combination with one or more antimicrobials. In the present study, all strains were found to be sensitive to polymyxin $\mathrm{B}$ and colistin

The lack of any new compounds in the near future indicates that national and local surveillance efforts are essential, to provide clinicians with correct information for choosing right antimicrobial therapy

In conclusion, the prevalence of $P$. aeruginosa infections in burn patients was found to be $41.79 \%$, and the prevalence of 
multi-drug resistance among these isolates was. Such a high prevalence of MDR $P$. aeruginosa infection in burn patients is a cause for concern because it poses a serious therapeutic challenge due to very limited treatment options. Restriction of 'selected antibiotic usage' and/or infection control policies must be tailored for each institution, to combat the rapid emergence of MDR $P$. aeruginosa in burn patients. Colistin still retains a high sensitivity and could, therefore, be used as a therapeutic alternative in case of multi-drug resistance (Puneet et al., 2015). The lack of newer antimicrobial agents with activities against $P$. aeruginosa, makes periodic studies on the antimicrobial resistance patterns very important (Babita et al., 2011).

\section{References}

Arslan, E., Dalay, C., Yavuz, M., Gocenter, L., Aearturk, S. 1999. Gram negative surveillance in burn patient. Ann. Burns Fire Disasters, XII: n2.

Asghar, A.H. 2012. Antimicrobial susceptibility and metallo $\beta$-lactamase production among Pseudomonas aeruginosa isolates from Makkah Hospital. Pak. J. Med. Sci., 28: 781-6.

Babita Sharma, Rajni Sharma, Nita Pal, Leela Vyas. 2011. Microbiological profile and antibiotic sensitivity pattern of burn wound infection in an Indian tertiary care hospital. J. Pure and Appl. Microbiol., 5(2): 837-841.

Ekrami, A., Kalantar, E. 2007. Bacterial infections in burn patients at a burn hospital in Iran. Indian J. Med. Res., 126: 541-4.

Indu Biswal, Balwinder Singh Arora, Dimple Kasana, Neetu Shree. 2014. Incidence of Multidrug Resistant Pseudomonas aeruginosa Isolated from Burn Patients and Environment of Teaching Institute. JCDR, 8(5): DC26-DC29.
Magiorakos, A.P. 2011. Multidrug Resistant (MDR), Extensively Drug Resistant (XDR) and Pandrug-1 Resistant (PDR) bacteria in healthcare settings. Expert Proposal for a Standardized International Terminology.

Moazami-Goudarzi, S., Eftekhar, F. 2012. Assessment of carbapenem susceptibility and multidrug resistance in Pseudomonas aeruginosa in burn isolates. Jundishapur J. Microbiol., 6: $162-5$

Naqvi, Z.A., Hasmi, K., Rizwan, Q.M., Kaarat, S.A. 2005. Multidrug resistance in Pseudomonas aeruginosa: A healthcare associated infectio threat in burn patient. Pak. J. Pharmacol., 22: 915.

Noha, A., Hassuna, Amany Hosney Ibrahim Mohamed, Sahar Mohamed AboEleunoon, Hazem Abdel-Wahab, A. Rizk. 2015. High Prevalence of Multidrug Resistant Pseudomonas aeruginosa recovered from Infected burn wounds in children. Arch. Clin. Microbiol., 6 No 4: 1.

Pier, G.B., Ramphal, R. 2010. Pseudomonas aeruginosa. In: Mandell GL, Bennett JE, Dolin R, editors. Principles and Practice of Infectious Diseases. 7th ed. Philadelphia: Churchill Livingstone Elsevier; p. 2835-60

Puneet Bhatt, Khushi Ram Rathi, Santanu Hazra, Alok Sharma, Vishal Shete. 2015. Prevalence of multidrug resistant Pseudomonas aeruginosa infection in burn patients at a tertiary care centre. Indian J. Burns, 23: 56-59.

Saderi, H., Falipour, H.L., Owalia, P., Salimi, H. 2014. Detection of metallo $\beta$ lactamase producing Pseudomonas aeruginosa isolated from burn patient in Tehran, Iran. Lab. Med., 41: 609-12.

Srinivasan, S., Vartak, A.M., Patil, A., Saldanha, J. 2009. Bacteriology of burn wound at the Baba Jerbai Wadia 
hospital for children, Mumbai- A 13 year study. Indian J. Plastic Surg., 42(2): 213-8.

Upadhaya, S., R. Shenoy, V. Shetty, A. Lamsal, P. Lamichhane, S. Pokhrel.
2014. Multi -drug Resistant Pseudomonas aeruginosa isolated from Intensive Care Burn Unit. Int. J. Biomed. Res., 5: 4.

\section{How to cite this article:}

Romika Dawra, Rajni Sharma, Rekha Bachhiwal and Aruna Vyas. 2017. High Incidence of Multidrug Resistant Pseudomonas aeruginosa Isolated from Infected Burn Wounds in a Tertiary Hospital. Int.J.Curr.Microbiol.App.Sci. 6(2): 1134-1139.

doi: http://dx.doi.org/10.20546/ijcmas.2017.602.128 